Massimiliano Signifredi

HTTPS://ORCID.ORG/OOOO-0002-3558-6I 58

Università Roma Tre

\title{
Visione messianica e realismo della storia in Giovanni Paolo II
}

\section{Abstract}

Paweł Rojek in his Liturgia dziejów [Liturgy of History] rightly pointed the importance of Polish messianism for the understanding of John Paul II. It is especially convincing when one consider the early poetry of the future Pope, the testimony of his personal secretary sister Emilia Ehrlich, and the importance of suffering, both in theological reflexion, and personal life of John Paul II. Nevertheless, papal messianism was a very special kind. First, the concept of nation, adopted by John Paul II, did not exclude the universal dimension of humanity. The pope applied the principles of his theology of nation not only for Poland, but also for many others nations, and in many occasions prised the multinational political formations. Secondly, for him, the missions of nations were connected rather with martyrdom than historical victories. Most importantly, John Paul II united the messianistic vision with a very realistic approach to the history. In result, his ideas turned out to be a fatal threat for communist regimes in Eastern Europe. The Solidarity movement in Poland, inspired by John Paul II, might be seen as an attempt of realisation of this specific messianistic vision.

KeYwORDs

John Paul II, theology of nation, theology of history, messianism, collapse of communism 


\section{IL RITORNO DEL MESSIANISMO}

Nel panorama degli studi dedicati a Karol Wojtyła, Liturgia dziejów. Jan Pawet II i polski mesjanizm [Liturgia della storia. Giovanni Paolo II e il messianismo polacco] si rivela un libro di grande interesse, la cui lettura invita a ulteriori analisi e approfondimenti ${ }^{\perp}$. Oggetto del lavoro di Paweł Rojek è un aspetto finora trascurato dagli studiosi di Giovanni Paolo II, ossia il suo rapporto con il filone romantico, mai sopito nelle menti e nei cuori dei polacchi, che assegna alla loro nazione una missione particolare nella storia del mondo. E ciò che va sotto il nome di «messianismo", un oggetto misterioso e affascinante, di cui talora affiorano manifestazioni più evidenti e che, a volte, viene osservato con imbarazzo o fastidio da alcuni polacchi, desiderosi di vivere in un paese «normale» ${ }^{2}$.

Una prima osservazione sul libro di Rojek non può non coglierne dunque il tempismo: Liturgia della storia arriva al momento opportuno, perché oltre a proporre un'interpretazione originale della personalità del polacco più noto del Novecento, aiuta a decifrare un fenomeno, di cui soprattutto negli ultimi anni si osserva la reviviscenza. In Polonia il messianismo è tornato di moda, e non solamente nelle aule universitarie. Alla categoria di messianismo si deve ricorrere, infatti, per spiegare la cosiddetta religione di Smoleńsk, sorta in seguito al disastro aereo del ro aprile 20Io, in cui persero la vita il presidente Lech Kaczyński e altre 95 persone. Questa tragedia, inserita «nella tradizione martirologica polacca, accanto al massacro di Katyń e alle numerose insurrezioni represse nel sangue», è divenuta il mito fondativo di un nuovo culto di massa ${ }^{3}$. Emblematiche a riguardo le dichiarazioni di Tomasz Terlikowski, già direttore di Telewizja Republika:

I P. Rojek, Liturgia dziejów. Jan Pawet II i polski mesjanizm, Kraków 2or6; cfr. P. Rojek, John Paul II and the Polish Messianism. Introduction to the Liturgy of History, transl. by K. Popowicz, "Theological Research" 7 (2019), p. 9-27.

2 Sul messianismo polacco esiste un'ampia bibliografia. Si vedano, tra i tanti titoli, A.Walicki,PhilosophyandRomaticNationalism:The CaseofPoland,Oxford I982;J.Plumyène, Le nazioni romantiche. Storia del nazionalismo nel XIX secolo, Firenze 1982, p. I69-187; P. Michel, Messianisme polonaise et histoire contemporaine, in: Le rêve de Compostelle. Vers la restauration d'une Europe chrétienne?, sous la direction de R. Luneau, P. Ladrière, Paris 1989, p. 52-67; P. Brian, Thy Kingdom Come: Patriotism, Prophecy, and the Catholic Hierarchy in Nineteenth-Century Poland, «The Catholic Historical Review» 2 (2003), p. 213-239.

3 C. Michalski, La religione di Smolensk, «Limes» I (20I4), p. 25-3I. 
Abbiamo tentato di sottrarci alla missione davanti alla quale ci ha posto Dio per trovare rifugio nella «normalità» dell'Occidente. Se è così, allora questa tragedia è un chiaro monito che non ci è permesso essere un popolo «normale» e che ogni tanto Dio Nostro Signore esige da noi un tributo di sangue ${ }^{4}$.

Un'ulteriore prova che in Polonia il messianismo gode di buona salute e ampio seguito a livello popolare è il successo del Rosario alle frontiere, che il 7 ottobre 2017 ha raccolto I milione di polacchi in circa 4000 punti lungo i confini del paese. Secondo i carismatici organizzatori dello spettacolare raduno, Maciej Bodasiński e Lech Dokowicz, «la potente preghiera del rosario [poteva] incidere sulle sorti della Polonia, dell'Europa e anche del mondo intero»5. Al di là delle controversie relative alla scelta della data - l'anniversario della battaglia di Lepanto - e al chiaro messaggio politico di chiusura all'Europa e ai migranti, il Rosario alle frontiere si adatta perfettamente, a mio avviso, alle caratteristiche delineate da Andrzej Kobyliński a proposito della «pentecostalizzazione» della Chiesa nel XXI secolo, ossia «la convinzione che il cristianesimo in Polonia - dopo il cosiddetto risveglio carismatico - abbia da giocare un'importante missione storica in relazione a tutto il resto del mondo» ${ }^{6}$. Analogamente Alfred Wierzbicki mette in guardia dalla massiccia diffusione dell'orgoglio nazionale accompagnato dalla rinascita del «sarmatismo», l'ideologia del XVI secolo che assegnava ai nobili polacchi una particolare missione in virtù della loro discendenza dalla leggendaria nazione dei sarmati ${ }^{7}$. Questi fenomeni vanno di pari passo con la fortuna che, nella Polonia di oggi, incontra

4 C. Michalski, La religione di Smolensk, op. cit., p. 30.

5 Mi permetto di rinviare al mio articolo, M. Signifredi, Cristo al servizio della Polonia, «Limes» 6 (2018), p. 235-242.

6 A. Kobyliński, Le dimensioni etiche dell'odierna pentecostalizzazione del cristianesimo, «Rivista Teologica di Lugano» 20 (2015), p. 219.

7 A. M. Wierzbicki, Present Condition and Role of the Catholic Church in Poland, «Occasional Papers on Religion in Eastern Europe» 38 (2018), p. 92-Io3. Cfr. anche L. Marinelli, Chi sono i polacchi?, «Limes» I (2014), p. 60: «Da mito etnogenico” del XV e XVI secolo, "il sarmatismo divenne in breve ideologia e vero e proprio stile di vita", le cui conseguenze più nefaste "furono una megalomania nazionale, un patriottismo religioso squillante, ma anche piuttosto superficiale, il gusto per il lusso e la sua esibizione"». 
la "politica storica», mirante a riscrivere la storiografia contemporanea secondo i canoni della «nazione immacolata»: per usare l'efficace definizione di Marcin Kula, si tratta di una «bistoria patriae, dal carattere sostanzialmente agiografico», legata a «una visione dalle tinte romantiche, missionarie, vittimistiche, incentrata su insurrezioni fallite ma in fondo vittoriose» ${ }^{8}$.

Per tornare a Liturgia della storia, Rojek non solo è consapevole del ritorno in auge del messianismo, ma è anche convinto che esso costituisca «una chiave interpretativa del pensiero di Giovanni Paolo II», notando che «la sua teologia della storia, la teologia della sofferenza e della nazione sono profondamente radicate nelle nostre idee romantiche»?. Si tratta indubbiamente di un'interpretazione destinata ad alimentare il dibattito, come mostrano le riserve espresse da diversi studiosi su $\mathrm{Li}$ turgia della stori ${ }^{\text {Io }}$. Senza entrare nel merito delle tesi di Rojek e dei suoi recensori, il libro si fa apprezzare per tre motivi.

Anzitutto Rojek ha il pregio di rileggere le poesie giovanili di Wojtyła, quasi completamente sconosciute, perché non incluse nella raccolta della Libreria Editrice Vaticana ${ }^{\text {II. }}$ Queste opere, considerate dallo stesso Wojtyła «artisticamente immature», sono state poi pubblicate in diverse edizioni generalmente poco reperibili ${ }^{\mathrm{r}}$. Lo stesso vale per l'epistolario tra il giovane Wojtyła e Mieczysław Kotlarczyk, una fonte assolutamente indispensabile, ma poco nota, per ricostruire l'itinerario intellettuale e spirituale di Giovanni Paolo $\mathrm{II}^{13}$. La prima produzione poetica wojtyliana, risalente al I939, presenta due tratti distintivi: il richiamo ai modelli romantici, assai in voga nel clima di rinascita della Polonia indipendente, e le cupe premonizioni della guerra, che di lì a poco avrebbe devastato l'Europa, proprio a partire dalla Polonia. Oltre che ricche di immagini significative, le poesie del giovane

8 M. Kula, La storia ad usum populi, «Limes» I (2014), p. 70-7I.

9 P. Rojek, Liturgia dziejów, op. cit., p. I4.

ıo Cfr. A. Draguła, Teologia narodu bez teologii, «Więź» 3 (2017), p. 226-232 e A. Wierzbicki, Kruche dziedzictwo. Jan Pawet II od nowa, Warszawa 2018, p. I5I-I54.

II K. Wojtyła, Tutte le opere letterarie. Poesie, drammi e scritti sul teatro, MilanoCittà del Vaticano 20or. Sulla tua bianca tomba e Magnificat sono le uniche opere giovanili a essere pubblicate in questa raccolta.

I2 K. Wojtyła, Le poesie giovanili (Salterio di Davide, Libro slavo, Salterio rinascimentale), Roma 2004, p. 23.

I3 K. Wojtyła, Il Teatro Rapsodico. Articoli e Lettere, Roma 2003. 
Wojtyła ci dicono molto sul futuro papa, sui temi che avrebbe affrontato, sulle battaglie che avrebbe combattuto. Andrea Riccardi ha osservato che alla poesia di Wojtyła non è stata dedicata «sufficiente attenzione»: «Tanto si è studiata la figura del papa, ma poco si è considerato il poeta o l'approccio da poeta e drammaturgo che caratterizzano Wojtyła» ${ }^{14}$.

Un secondo aspetto che rende prezioso il lavoro di Rojek è il recupero di Osservazioni su alcuni aspetti del messianismo, una relazione tenuta nel I982 da suor Emilia Ehrlich nella casa per i pellegrini polacchi sulla Via Cassia. In questo testo, completamente dimenticato, la suora orsolina - per anni segretaria e bibliotecaria di Wojtyła - esamina i testi di alcuni messianisti polacchi, a cominciare da Stanisław Orzechowski (sec. XVI), mettendoli a confronto con le idee del Concilio Vaticano II. Questa analisi si basa su una lettura puntuale di Alle fonti del rinnovamento, volume pubblicato nel 1972 dall'allora cardinale Wojtyła, la cui edizione italiana fu tradotta e curata dalla stessa suor Emilia ${ }^{15}$. La relazione, che individua una sostanziale continuità tra il messianismo polacco e il Concilio che si rivolgeva al "popolo messianico», si conclude così:

Lideale messianico oggi non è affatto meno esigente, che ai tempi di Mickiewicz. Ma insieme a questi compiti enormi riceviamo sia la forza per realizzarli, sia grazie così grandi, che Dio concede alle nazioni una volta ogni mille annir ${ }^{16}$.

Difficile resistere alla tentazione di pensare che queste considerazioni non fossero, almeno in parte, condivise dal papa polacco, che ogni giorno aveva modo di scambiare qualche parola con suor Emilia.

I4 A. Riccardi, Giovanni Paolo. La biografia, Cinisello Balsamo 2oII, p. 94-95. R. Buttiglione, Il pensiero dell'uomo che divenne Giovanni Paolo II, Milano I998, p. 4I-5I e 274-28I, illustra la formazione letteraria di Wojtyła e nota il particolare ruolo sociale dei poeti in Polonia: «L'opera d'arte è concepita nella tradizione polacca come formatrice dell'ethos della nazione; è attraverso di essa che il valore universale riceve concretezza ed evidenza emozionale».

I5 K. Wojtyła, Alle fonti del rinnovamento. Studio sull'attuazione del Concilio vaticano secondo, Città del Vaticano I98I.

I6 E. Ehrlich, Remarks on Some Aspects of Messianism, transl. by K. Popowicz, "Theological Research" 7 (2019), p. 49. 
In terzo luogo, Liturgia della storia accosta alla mistica di suor Faustina Kowalska la riflessione di Wojtyła sulla sofferenza, un cardine della sua spiritualità e della sua stessa esistenza. «Giovanni Paolo II non è stato solo un teorico della sofferenza», scrive Rojek, incrociando le sue tormentate vicende biografiche nella Cracovia occupata dai nazisti con le opere composte in quegli annir ${ }^{17}$. A riguardo Benedetto XVI ha osservato che il suo precedessore «veniva da un popolo sofferente, quello polacco, sottoposto a tante prove nella sua storia. Da questo popolo sofferente, dopo tante persecuzioni, si sviluppa la forza di sperare ${ }^{18}$.

In questo articolo mi soffermerò sull'idea di nazione di Wojtyła, sulla sua concezione della storia, concludendo con qualche osservazione sul suo contributo alla fine del comunismo.

\section{Giovanni Paolo II, polacco}

Per Wojtyła la «patria» era anzitutto la «sua» Polonia, verso la quale aveva un legame filiale. Aldino Cazzago ha osservato che Giovanni $\mathrm{Pa}-$ olo II «non si è limitato a vivere una sorta di passiva osmosi con quanto la sua patria stava vivendo», ma ha compiuto «un originale lavoro di riflessione sul "soggetto" della "patria", sulla sua identità e sul ruolo della Chiesa in essa» $»^{19}$. Per Wojtyła «nel concetto stesso di patria, è contenuto un profondo legame tra l'aspetto spirituale e quello materiale, tra la cultura e il territorio ${ }^{20}$. Evidente quindi la sua relazione affettiva con la Polonia. «La Cracovia vecchia e nuova - scrisse ai pellegrini della sua ex arcidiocesi dieci giorni dopo l'elezione - costituisce uno strato della mia anima che non posso lasciare. Lo strato della mia esperienza, della mia fede, del mio amore $»^{2 \mathrm{r}}$.

«Io, Giovanni Paolo, figlio della Nazione polacca», esclamò a Compostela nel 1982. Questa espressione ritorna in uno dei discorsi più

17 Cfr. P. Rojek, Liturgia dziejów, op. cit., p. I66-198.

I8 Cfr. A. Riccardi, Giovanni Paolo II, op. cit., p. I89.

I9 A. Cazzago, Giovanni Paolo II. «Ama gli altri popoli come il tuo», Milano 2013, p. 9-Io.

20 Giovanni Paolo II, Memoria e identità. Conversazioni a cavallo dei millenni, Milano 2005, p. 77.

21 Giovanni Paolo II, All'Arcidiocesi di Cracovia, 23 ottobre 1978, in: Insegnamenti di Giovanni Paolo II, Vol. r: 1978, Città del Vaticano 1979, p. 56-57. 
intensi del lungo pontificato, quello all'Unesco, che rivela tanto del pensiero di Wojtyła:

Io sono figlio di una nazione, che ha vissuto le più grandi esperienze della storia, che i suoi vicini hanno condannato a morte a più riprese, ma che è sopravvissuta e che è rimasta se stessa. Essa ha conservato la sua identità e [...] la sua sovranità nazionale, non appoggiandosi sulle risorse della forza fisica, ma unicamente appoggiandosi sulla sua cultura $^{22}$.

Wojtyła accentuava la sua provenienza, a differenza dei suoi predecessori. Ha osservato in proposito Riccardi che

nel pontificato romano, tradizionalmente, tutto tende a rimuovere l'origine nazionale del papa, «padre comune». I predecessori di Giovanni Paolo II tendevano quasi a spersonalizzarsi e spogliarsi della loro identità, «snazionalizzandosi», pur non perdendo interesse per le questioni nazionali. Pio XII, ricevendo gli italiani, parlava di «vostra patria». $\mathrm{Pa}-$ olo VI, pur avendo compiuto vari viaggi in Italia da papa, non era mai tornato da papa nella natia Brescia né nell'arcidiocesi di Milano ${ }^{23}$.

Giovanni Paolo II era e si diceva polacco, anche contro il protocollo, fino a firmare la lettera apostolica Rutilans Agmen del r979: «Giovanni Paolo II, polacco» ${ }^{24}$.

La sottolineatura della sua origine nazionale appariva strana in Occidente, a cominciare dall'Italia, la cui cultura, in anni di contrapposizione ideologica, era quasi allergica alla parola «patria» per l'abuso che ne era stato fatto dal fascismo. Probabilmente Giovanni Paolo II ne era consapevole e per questo approfittava di ogni occasione per illustrare questa sua visione originale, come per evitare che venisse confusa con simpatie nazionalistiche. «Non voglio essere accusato da qualcuno

22 Giovanni Paolo II, L'allocuzione all'Organizzazione delle Nazioni Unite per l'Educazione, la Scienza e la Cultura, 2 giugno I980, in: Insegnamenti di Giovanni Paolo II, Vol. 3/r: 1980, Città del Vaticano I981, p. 1636-1655.

23 A. Riccardi, Giovanni Paolo, op. cit., p. 30.

24 Giovanni Paolo II, Lettera apostolica Rutilans Agmen alla Chiesa di polonia nel IX Centenario del martirio di san Stanislao, 8 maggio 1979, in: Insegnamenti di Giovanni Paolo II, Vol. 2/r: 1979, Città del Vaticano 1979, p. I095-IIor. 
di patriottismo locale. Devo farci molta attenzione, soprattutto dopo il i6 ottobre dell'anno scorso", confidò Wojtyła in un discorso «informale e improvvisato» - non riportato nei volumi degli Insegnamenti a un gruppo di superiori degli ordini religiosi maschili a Częstochowa durante il suo primo viaggio in Polonia nel $1979^{25}$. Al primo incontro con gli ambasciatori accreditati presso la S. Sede Giovanni Paolo II osservò: «La storia della mia patria d'origine $\mathrm{mi}$ ha insegnato a rispettare i valori specifici di ogni nazione, di ogni popolo, le loro tradizioni e i loro diritti tra gli altri popoli $»^{26}$. Ma anche nella Polonia popolare, esauritasi la via nazionalcomunista di Władysław Gomułka, l'insistenza di Wojtyła sul concetto di «patria» poteva suonare anacronistica, se non sospetta agli orecchi dei governanti socialisti, abituati a servirsi del patriottismo esclusivamente a fini propagandistici. Significativa è la spiegazione offerta da Giovanni Paolo II a Gierek, in occasione del loro incontro a Varsavia il 2 giugno 1979:

La parola "patria» ha per noi un tale significato, concettuale ed insieme affettivo, che le altre Nazioni dell'Europa e del mondo sembra non lo conoscano, specialmente quelle che non hanno sperimentato - come la nostra Nazione - danni storici, ingiustizie e minacce ${ }^{27}$.

\section{Per essere nazionale, essere sovra-NAZionale}

Ma qual era il modello di nazione di Giovanni Paolo II? Ha scritto Roberto Morozzo della Rocca: «Più Wojtyła era polacco e più era europeo e universale. Wojtyła pensava che proprio il radicamento in una cultura nazionale rendesse possibile aprirsi a comunità più ampie» ${ }^{28}$. Da Norwid, il suo poeta preferito, trasse una citazione illuminante:

25 Jan Paweł II, Pielgrzymki do Ojczyzny. Przemówienia, homilie, Kraków 2012, p. 57. L'incontro è menzionato di sfuggita in L'attività della Santa Sede nel 1979, Città del Vaticano I980, p. 384 .

26 Giovanni Paolo II, Al Corpo diplomatico, 20 ottobre 1978, in: Insegnamenti di Giovanni Paolo II, Vol. I: 1978, Città del Vaticano 1979, p. 27.

27 Giovanni Paolo II, L'incontro con le autorità civili, 2 giugno 1979, in: Insegnamenti di Giovanni Paolo II, Vol. 2/1: 1979, Città del Vaticano 1979, p. I38o.

28 R. Morozzo della Rocca, Tra Est e Ovest. Agostino Casaroli diplomatico vaticano, Cinisello Balsamo 20I4, p. 27 I. 
Per essere nazionale - essere sovra-nazionale!

E per essere umano, per questo essere sovraumano... essere doppio e uno - perché? ${ }^{29}$

Insomma in Wojtyła, animo slavo che sapeva convivere con le antinomie, le due dimensioni - nazionale e sovranazionale - si tenevano insieme senza contraddizioni ${ }^{30}$. Non è un caso che della storia polacca Giovanni Paolo II prediligesse l'epoca jagellonica, la Polonia tollerante e inclusiva dove coabitavano polacchi, ebrei, lituani, ucraini, bielorussi. L'eredità jagellonica era sentita con orgoglio da Wojtyła, che amava ricordare figure come Paweł Włodkowic e ripetere il detto di Sigismondo Augusto al tempo delle guerre di religione: «Non sarò il re delle vostre coscienze». In Memoria e identità Giovanni Paolo II osservò con evidente dispiacere:

Lo spirito polacco è, in fondo, la molteplicità e il pluralismo, e non la ristrettezza e la chiusura. Sembra tuttavia che la dimensione «jagellonica» dello spirito polacco, ora ricordata, abbia purtroppo cessato di essere qualcosa di ovvio nel nostro tempo ${ }^{31}$.

Per allargare lo sguardo oltre alla Polonia, è interessante notare l'apprezzamento di Giovanni Paolo II per grandi Stati multietnici, come il Canada e il Brasile, ma anche la difesa caparbia, e poco sostenuta dalla comunità internazionale, di paesi più piccoli, le cui diverse componenti etniche avevano cessato di coabitare in pace sprofondando in terribili guerre civili, come il Libano e la Jugoslavia.

Il secondo elemento dell'identità nazionale polacca accentuato da Giovanni Paolo II era la sua storia di sofferenza, in particolare nel $\mathrm{XX}$ secolo, che l'aveva preparata ad affrontare grandi sfide. Una settimana dopo l'elezione a papa, nella Lettera ai connazionali, Wojtyła affermò

29 C. K. Norwid, Rzecz o wolności stowa, in: C. K. Norwid, Pisma wszystkie, Warszawa 1971, Vol. 3: Poematy, p. 564, cfr. Giovanni Paolo II, Ai rappresentanti dell'Istituto del patrimonio nazionale polacco, degli scienziati e dei cultori del poeta polacco Cyprian $\mathrm{Ka}$ mil Norwid, r luglio 200I, in: Insegnamenti di Giovanni Paolo II, Vol. 24/r: 200I, Città del Vaticano 2002, p. 7 .

30 Importanti osservazioni sull'animo slavo di Wojtyła in R. Morozzo della Rocca, Giovanni Paolo II papa slavo, «Sanctorum» 3 (2006), p. 55-59.

3I Giovanni Paolo II, Memoria e identità, op. cit., p. Io9. 
che la Chiesa in Polonia recava «una particolare testimonianza alla quale tutto il mondo guarda[va] $\aleph^{3^{2}}$. Nel citato incontro coi superiori degli ordini religiosi maschili a Częstochowa, Wojtyła osservò che «le esperienze assai difficili» trascorse dai polacchi nella storia permettevano loro di leggere «i segni dei tempi, diversamente e forse più acutamente» rispetto agli europei occidentali. Questa differenza non era «un apriorismo, ma un aposteriorismo (sic) teologico e pastorale o, se si vuole, un empirismo» ${ }^{33}$. Insomma la missione affidata dalla Provvidenza ai polacchi, per Wojtyła, non era solamente, e semplicemente, una convinzione religiosa, filosofica o letteraria. Essa maturò nella vita a Cracovia durante l'occupazione nazista. Considerandosi un sopravvissuto della guerra e meditando sulla spiritualità di san Luigi Grignon de Monfort, Wojtyła si sentì investito di una grande missione. Appena quattro giorni dopo l'elezione, scrisse all'amica Wanda Półtawska:

Da oltre vent'anni, da quando Andrzej mi disse per la prima volta: "Duśka è stata a Ravensbück», è nata nella mia consapevolezza la convinzione che Dio mi dava e mi assegnava te, affinché in un certo senso io «compensassi» quello che avevi sofferto lì. E ho pensato: lei ha sofferto al mio posto. A me Dio ha risparmiato quella prova, perché lei è stata lì. Si può dire che questa convinzione fosse «irrazionale», tuttavia essa è sempre stata in me - e continua a esserci ${ }^{34}$.

Queste espressioni tornano nell'autobiografico Dono e mistero in riferimento a Jerzy Zachuta, compagno di Wojtyła al seminario clandestino di Cracovia, arrestato e fucilato dalla Gestapo. Nella dedica del libro ai nonni paterni, Rojek inserisce un riferimento personale significativo, ricordando di aver ascoltato da loro diversi racconti sul parroco della loro cittadina, Andrzej Zachuta, fratello di Jerzy, con cui Wojtyła era rimasto in amicizia, visitandolo spesso anche da arcivescovo di Cracovia $^{35}$. Giovanni Paolo II avvertiva un «debito» nei confronti dei tanti amici scomparsi durante la guerra:

32 Giovanni Paolo II, Ai pellegrini polacchi, 23 ottobre 1978, in: Insegnamenti di Giovanni Paolo II, Vol. r: 1978, Città del Vaticano 1979, p. 52.

33 Jan Pawet II na ziemi polskiej, red. S. Dziwisz i in., Watykan I979, p. 79-8o.

34 W. Półtawska, Diario di un'amicizia. La famiglia Póttawski e Karol Wojtyta, Cinisello Balsamo 2010, p. 403-404.

35 P. Rojek, Liturgia dziejów, op. cit., p. 26-27. 
Del grande e orrendo theatrum della seconda guerra mondiale mi fu risparmiato molto. Ogni giorno avrei potuto essere prelevato dalla casa, dalla cava di pietra, dalla fabbrica per essere portato in campo di concentramento. A volte mi domandavo: tanti miei coetanei perdono la vita, perché non io? Oggi so che non è un caso ${ }^{36}$.

La mistica di Giovanni Paolo II era attraversata da una dimensione agonica: alla vigilia del Grande Giubileo del 2000 invitò la Chiesa a meditare sulla memoria dei martiri contemporanei e volle che la basilica di San Bartolomeo all'Isola Tiberina a Roma, affidata alla Comunità di Sant'Egidio, fosse loro dedicata ${ }^{37}$. Il tema del martirio era centrale nella spiritualità del papa polacco. Come rivelò a André Frossard, citando Giobbe, la vita dell'uomo era una lotta: «L'uomo deve ogni giorno affrontare il male e lottare per il bene. Il vero bene morale non è facile: bisogna conquistarlo continuamente in se stessi, negli altri, nella vita sociale e internazionale» $3^{8}$.

\section{TutTi I POPOLI SONO BUONI}

A questa dimensione agonica si accompagnava una mistica geografica: «Ogni giorno, cè una geografia spirituale che percorro. La mia spiritualità è un po' geografica», confidava ${ }^{39}$. Era il senso dei viaggi, strumento del suo pontificato davvero globale, che permettevano al papa di entrare in contatto diretto con le nazioni.

La nazione veniva prima dello Stato e la parola stessa entrò in permanenza nel linguaggio papale, lasciando perplessa l'opinione pubblica occidentale. Per Wojtyła la nazione era «la grande comunità degli

36 Giovanni Paolo II, Dono e mistero. Nel $50^{\circ}$ del mio sacerdozio, Città del Vaticano I996, p. 45 e 47 .

37 Cfr. A. Riccardi, Il secolo del martirio. I cristiani nel Novecento, Milano 2000.

38 Non abbiate paura! André Frossard dialoga con Giovanni Paolo II, Milano I983, p. 264 .

39 Testo citato da L. Accattoli, D. Del Rio, Wojtyta. Il nuovo Mosè, Milano I988, p. I26. Si veda anche M. Mokrzycki, B. Grysiak, I martedì di Karol. La vita quotidiana di Giovanni Paolo II, Cinisello Balsamo 2013, p. 63: «Una volta disse: Io pregherò per tutto il mond. E poi a cena cominciò a menzionare le nazioni, le repubbliche dell'Africa e dell'Asia. Pensai che davvero pregasse per tutto il mondo». 
uomini che sono uniti da diversi legami, ma soprattutto, precisamente, dalla cultura». Il papa sviluppò una teologia delle nazioni, per cui ognuna - anche se piccola - aveva una funzione storica e provvidenziale ${ }^{40}$. Riecheggiano le parole del patriarca di Costantinopoli Athenagoras, che in un libro-intervista del ig68 con Olivier Clément, aveva detto: "Ho conosciuto bene gli slavi. Ho anche osservato i tedeschi e gli austriaci. Con i francesi ho vissuto due anni. Tutti i popoli sono buoni. Ognuno merita rispetto e ammirazione ${ }^{4 \mathrm{~T}}$.

In fondo la riflessione di Wojtyła sui concetti di patria e nazione non era dissimile da quella che in Raggi di paternità egli svolse sull'incessante cammino dell'umanità «dal polo della solitudine al polo dell'amore»" Secondo Tischner la chiave di Raggi di paternità era «l'idea dell'integrazione creativa delle persone», che si poteva riassumere con le parole: "grazie a te io divento me stesso e grazie a me tu divieni te stesso» ${ }^{43}$. Parafrasando queste espressioni, si potrebbe dire che per Wojtyła non esisteva nazione senza le nazioni vicine. Non a caso, Giovanni Paolo II amava ricordare queste parole di Norwid: «La nazione è fatta non solo di quello che la distingue dalle altre, ma di quanto alle altre la unisce» ${ }^{44}$.

Per Wojtyła ogni nazione era importante. «Ogni uomo, ogni Nazione, ogni cultura e civiltà hanno un proprio ruolo da svolgere e un proprio posto nel misterioso piano di Dio e nell'universale storia della salvezza», scrisse nell'enciclica Slavorum apostoli ${ }^{45}$. "Ogni nazione ha la sua particolare vocazione, attraverso le tragiche vicissitudini della storia, ad incarnare un aspetto particolare della rivelazione del Verbo", aveva notato intervenendo a un convegno dedicato a Vjaceslav Ivanov, il filosofo russo avvicinatosial cattolicesimo senza abiurarel'ortodossia, morto a Roma nel $1949^{46}$.

40 Cfr. A. Riccardi, Giovani Paolo II, op. cit., p. 337.

4I Atenagora, O. Clément, Umanesimo spirituale. Dialoghi tra Oriente e Occidente, Cinisello Balsamo 20I3, p. 82.

42 K. Wojtyła, Tutte le opere letterarie, op. cit., p. 942.

43 K. Wojtyła, Tutte le opere letterarie, op. cit., p. 879.

44 C. K. Norwid, Znicestwienie Narodu, in: C. K. Norwid, Pisma wszystkie, t. 7: Proza, Warszawa 1973, p. 86, cfr. Giovanni Paolo II, Ai rappresentanti dell'Istituto del patrimonio nazionale polacco, op. cit., p. 8.

45 Giovanni Paolo II, Lettera enciclica «Slavorum apostoli», 2 giugno 1985, no. I9.

46 Giovanni Paolo II, Ai partecipanti ad un simposio internazionale su «Ivanov e la cultura del suo tempo", 28 maggio 1983, in: Insegnamenti di Giovanni Paolo II, Vol. 6/r: I983, Città del Vaticano I983, p. I380. 
Molti sono gli esempi di questa valorizzazione delle differenti vocazioni nazionali. Solo un cenno all'Italia, a cui Giovanni Paolo II riservò grande attenzione, nutrendo e realizzando il disegno di «un cattolicesimo di popolo» in grado di incidere nella vita nazionale. Il papa polacco difese l'unità d'Italia, minacciata negli anni '9o dalle spinte secessioniste della Lega Nord. In un luogo decisivo della sua geopolitica spirituale, Assisi, Wojtyła convocò una grande preghiera per l'Italia, per ricordarne la vocazione ${ }^{47}$.

Visitando Vienna nel 1983, definì il passato imperiale dell'Austria «un modello e uno specchio per l'Europa», perché aveva mostrato «in modo esemplare come una varietà di popoli [potesse] convivere in uno spazio ristretto, non senza problemi, in modo creativo, trovando nella molteplicità un'unità» ${ }^{4}$. Si ripresentava qui l'ammirazione di Wojtyła per gli Stati multietnici, come era l'Impero asburgico, a cui peraltro egli era legato perché il padre aveva servito nell'esercito austriaco.

La Francia era un paese decisivo nella geografia di Giovanni Paolo II. Nel corso del primo viaggio ricordò il battesimo di Clodoveo $\mathrm{e}$, senza essere capito in un paese segnato dalla laicità, propose l'orgoglio di essere cristiani ${ }^{49}$. All'aeroporto di Le Bourget, il I ${ }^{\circ}$ giugno I980, dopo aver elencato i santi francesi che lo avevano influenzato - da Giovanna d'Arco al padre de Foucauld, passando per Giovanni Maria Vianney e Bernadetta di Lourdes - affermò che attraverso di loro «la storia della salvezza entra[va] nella storia dei popoli, delle nazioni, delle patrie, dei continenti s $^{\circ}$. I santi erano "sempre gli uomini e le donne di "domani"», esclamò a Lisieux ${ }^{51}$.

47 Cfr. M. Impagliazzo, Giovanni Paolo II e l'Italia, in: I cattolici ele Chiese cristiane durante il pontificato di Giovanni Paolo II (1978-2005), a cura di E. Guerriero, M. Impagliazzo, Cinisello Balsamo 2006, p. 97-I24.

48 Giovanni Paolo II, La celebrazione dei Vespri nella Heldenplatz, Io settembre I983, in: Insegnamenti di Giovanni Paolo II, Vol. 6/2: 1983, Città del Vaticano 1984, p. 436-444.

49 Cfr. A. Riccardi, Giovanni Paolo II, op. cit., p. 243

50 Giovanni Paolo II, L'omelia della messa a Le Bourget, I giugno I980, in: Insegnamenti di Giovanni Paolo II, Vol.3/r: I980, Città del Vaticano 1980, p. 1585-1592.

5I Giovanni Paolo II, L'omelia della messa a Lisieux, 2 giugno 1980, in: Insegnamenti di Giovanni Paolo II, Vol. 3/r: 1980, Città del Vaticano I980, p. I659. 
Il tema della santità si legava a quello del martirio, che - come ho già detto - era uno dei fondamenti della mistica di Giovanni Paolo $\mathrm{II}^{52}$. Sempre nel r980 il papa andò ad Otranto, estremo lembo meridionale della Puglia, per ricordarne i martiri nel cinquecentenario ma volse gli occhi verso la «non distante eroica Chiesa in Albania, sconvolta da dura e prolungata persecuzione ma arricchita dalla testimonianza dei suoi martiri». Per Giovanni Paolo II, occorreva «restare in comunione coi martiri», perché essi «scava[va]no l'alveo profondo nel fiume divino della storia»33. Attraverso i santi si capisce la storia delle nazioni, affermò Wojtyła. Di qui la sua insistenza per festeggiare anniversari significativi per i paesi dell'Europa centro-orientale lungo gli anni Ottanta: san Casimiro in Lituania, san Metodio in Cecoslovacchia e Bulgaria, santo Stefano in Ungheria, il battesimo di san Vladimiro in Russia e Ucraina, per ricordare alcune delle date di quella che Bernard Lecomte ha definito "guerra di anniversari» ${ }^{54}$.

\section{IL PAPA SLAVO E L'EST COMUNISTA}

La sfida di Giovanni Paolo II fu avvertita come una minaccia dal potere comunista in Europa centro-orientale e nella stessa Unione Sovietica, come dimostra in modo evidente la documentazione degli archivis5. Particolarmente temuta era la possibilità di un contagio dalla Polonia agli altri paesi satelliti e alle repubbliche sovietiche. Se già all'inizio del pontificato non erano mancati segnali preoccupanti - il papa ricevette dissidenti ucraini e lituani - fu il primo viaggio in Polonia ad allarmare i sovietici, con il noto discorso di Gniezno sulla vocazione del «papa slavo» volta a manifestare «l'unità spirituale dell'Europa». L'insistenza del papa sulla «religiosità delle nazioni slave», per il ministro per gli Affari religiosi polacco Kazimierz Kąkol, indicava che Wojtyła

52 Cfr. A. Roccucci, Senso della storia e tensione escatologica. Giovanni Paolo II e la santità di fronte alla crisi della modernità, "Sanctorum» 3 (2006), p. 15-30.

53 Giovanni Paolo II, L'omelia sul colle dei martiri, 5 ottobre 1980, in: Insegnamenti di Giovanni Paolo II, Vol. 3/2: 1980, Città del Vaticano I98I, p. 777.

54 B. Lecomte, La verità prevarrà sempre sulla menzogna. Come papa Giovanni Paolo II ha sconfitto il comunismo, Milano 1992, p. I53-154.

55 Cfr. il mio libro, M. Signifredi, Giovanni Paolo II e la fine del comunismo. La transizione in Polonia (1978-I989), Milano 2013. 
inaugurava «un'offensiva finora non esercitata dal Vaticano» ${ }^{56}$. Nel luglio 1979, i segretari di II partiti comunisti dell'Est, riuniti a Berlino, misero in guardia contro la minaccia del «nazionalismo» e del "panslavismo", ravvisando un parallelismo tra la vicenda polacca e quella iraniana. Un'osservazione interessante, perché ideologi marxisti che avevano per decenni predetto la fine delle religioni percepirono i primi sintomi di quel risveglio, che molto più tardi gli studiosi occidentali avrebbero definito «rivincita di Dio» ${ }^{57}$. Nell'ottobre 1979, in un convegno di ministri per gli Affari religiosi dei paesi socialisti, sempre Kąkol osservò che Wojtyła lavorava per la «rinascita dei sentimenti e degli atteggiamenti religiosi nei paesi socialisti», mirante al «risveglio dei nazionalismi: lituano, ucraino, polacco, croato, slovacco, ceco». Kąkol rifletteva sul «ruolo speciale» della Chiesa polacca nell'architettura della nuova politica orientale wojtyliana e si soffermava sui pericoli rappresentati dalla «concezione messianica del panslavismo cristiano». Tenuto conto che tutti i popoli slavi erano compresi nel campo socialista, il progetto di Giovanni Paolo II appariva agli ideologi comunisti visionario, ma non meno pericoloso. La conclusione di Kąkol era che il Vaticano puntava alla «destabilizzazione politica» dei paesi socialisti: la religione aveva «il ruolo di un cavallo di Troia $\aleph^{8}$. Sono ragionamenti segnati da categorie ideologiche d'altri tempi, che nondimeno permettono di comprendere il sospetto del mondo comunista nei confronti di Giovanni Paolo II.

Quanto questi timori fossero fondati e quanto i polacchi fossero stati infiammati da Wojtyła è ben evidente nella nascita di Solidarność e nelle vicende che, nel giro di un decennio, avrebbero portato alla transizione pacifica del 1989 e, con essa, alla fine del comunismo in Europa. Non è possibile ripercorrere questa storia, ma vorrei soffermarmi sull'Appello ai lavoratori dell'Europa orientale del congresso di Solidarność, perché vi si rinviene, a mio avviso, l'apice del filone messianico nella Polonia degli anni Ottanta. Si tratta di una breve mozione che

56 Giudizio preliminare sullo svolgimento della visita di Giovanni Paolo II in Polonia (periodo 2-5/6/79), anonima ma di Kazimierz Kąkol, Archiwum Akt Nowych w Warszawie, Urząd do Spraw Wyznań w Warszawie, I37/r8, k. 62-68.

57 Cfr. G. Kepel, La Revanche de Dieu. Chrétiens, juifs et musulmans à la reconquête du monde, Paris I99I.

58 La politica del Vaticano nel momento attuale (progetto di intervento), Archiwum Akt Nowych w Warszawie, Urząd do Spraw Wyznań w Warszawie, 136/24, k. 16-25. 
fu approvata per acclamazione, tra l'euforia dei delegati al congresso e lo sconcerto di quegli intellettuali, come Bronisław Geremek e Jacek Kuroń, che fungevano da consiglieri del sindacato. Due giorni dopo Leonid Brežnev si infuriò per «questo documento pericoloso e provocatorio» che mirava a "creare confusione nei paesi socialisti e fomentare ogni sorta di gruppi di dissidenti» ${ }^{59}$. Redatto da Jan Lityński, un ex attivista del KOR, e letto al congresso da Andrzej Gwiazda - la sua lunga barba e il suo lento incedere verso il microfono rammentarono a un

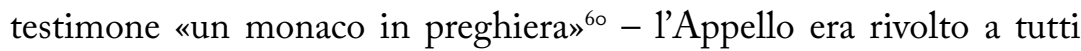
i paesi dell'Est, nominati uno per uno, e «a tutte le nazioni dell'URSS». Consapevoli di una «comunità di destini» fra i popoli di quella parte d'Europa, i polacchi invitavano i lavoratori dell'Est a diffidare delle «bugie» diffuse nei loro paesi a proposito di Solidarność e promettevano sostegno a chiunque intendesse «intraprendere il difficile percorso di lotta per i sindacati liberi» ${ }^{61}$.

Per quanto l'Appello non avesse alcuna possibilità di raggiungere i suoi destinatari, non ne va sottovalutato l'impatto emotivo. Tornano alla mente le espressioni del Libro dei pellegrini polacchi, in cui Mickiewicz scriveva che per un polacco la patria si trovava «in qualunque parte d'Europa la libertà [era] conculcata e si lottava per essa» ${ }^{62}$. Come notato da Walicki, Mickiewicz si era fatto portavoce di un'idea nazionale libera dalle passioni egoistiche e xenofobiche, concependo «un internazionalismo programmatico», che chiedeva sacrifici «in nome della causa comune di tutte le nazioni» ${ }^{6}$.

L’Appello rivelava il volto messianico di Solidarność, la sua aspirazione ad andare oltre la realtà polacca e proporsi come modello per altre nazioni sottomesse. Appariva velleitario ai governi occidentali, inquieti per le conseguenze dell'avventura polacca. Solidarność «scadeva

59 Lo sconcerto di Brežnev alla seduta del politbjuro del Partito Comunista dell'Unione Sovietica del ro settembre I981, citato da Soviet Deliberations during the Polish Crisis, 1980-1981, ed. M. Kramer, Washington I999, p. I37.

6o A. Kemp-Welch, Poland under Communism. A Cold War History, Cambridge 2008, p. 32I.

6I From Solidarity to Freedom. International Conference, Warsaw-Gdańsk, August 29-3I, 2005, Warsaw-Gdańsk 2005, p. 4.

62 A. Mickiewicz, Scritti politici, Torino 1965, p. Ior.

63 A. Walicki, Adam Mickiewicz and Polish Romantic Messianism, «Dialogue and Universalism» $3-4$ (2000), p. I74. 
nell'opinione dell'Occidente»: così all'indomani del congresso sindacale mons. Andrzej Deskur, confidente di Giovanni Paolo II ed esperto di mass media, riferì a mons. Bronisław Dąbrowski, segretario dell'episcopato polacco, le preoccupazioni dell'entourage di Wojtyła ${ }^{64}$. Di esso faceva parte anche Tischner, che intervenne al congresso di Solidarność. Nelle sue omelie durante le celebrazioni per i delegati e in un intenso discorso in aula, il prete filosofo rielaborò il pensiero di Norwid, parlando dell'etica del lavoro, ma anche dell'idea del compromesso come condizione fondamentale per un autentico dialogo. Tra l'altro, propose di allontanare lo «spirito di maligna sospettosità», che divideva "gli uomini in buoni e cattivi» e invitò "a stringere la mano anche all'avversario qui presente ${ }^{65}$. Il compromesso era la ricetta fondamentale indicata da Tischner: esso aveva reso esemplare per il mondo intero la pur breve esistenza di Solidarność. Ed in sé, il metodo del compromesso «metteva in discussione la tradizione romantica secondo cui una sconfitta eroica diventa[va] una vittoria spirituale» ${ }^{66}$.

Giovanni Paolo II è stato un visionario, che ha sperato di poter aprire varchi nei muri e ha messo in moto dinamiche inaspettate, forzando situazioni che sembravano bloccate e davanti a cui in molti, forse tutti, si erano rassegnati. In una poesia dell'autunno 1939 scrisse:

Vedi, madre - occorre in qualche luogo sfondare il grande portone, sbarrato, e occorre rompere la porta potente verso il Chiarore. Ogni giorno nelle chiese di quercia occorre desiderare e sentire come il canto si abbassa alle nostre corde. Occorre costruire le chiese personalmente ${ }^{67}$.

64 Cfr. P. Raina, Arcybiskup Dąbrowski. Rozmowy watykańskie, Warszawa 200I, p. 236.

65 J. Tischner, Guarire il lavoro ammalato, in: J. Tischner, Etica della solidarietà e del lavoro, trad. A. Setola, Castel Bolognese 2010, p. I47.

66 A. Kemp-Welch, Poland under Communism, p. 321. Cfr. anche J. Plumyène, Le nazioni romantiche, op. cit., p. 286: «Nella storia del nazionalismo le sconfitte sono momenti necessari. Riattivano nel presente la grande sconfitta mitica che ogni nazione pretende di aver subito in un leggendario altroquando. Sconfitte fondatrici, chiamate a vittoriose rivincite».

67 ...E quando Davide giunse alla sua terra madre, in: K. Wojtyła, Le poesie giovanili, op. cit., p. I68-I69. 
Convinto che le grandi svolte della storia si decidevano nel cuore dell'uomo, Wojtyła è riuscito a convivere con le antinomie: visione messianica e realismo della storia erano complementari in un disegno di liberazione. Ha lavorato per l'evoluzione del sistema, perché si potesse aprire verso la libertà e per questo non ha disdegnato di dialogare con Jaruzelski e Gorbačëv. E dopo la fine del comunismo non ha condiviso i toni trionfalistici della vittoria dell'Occidente capitalista. Anzi, alieno per indole a qualsiasi protagonismo - le sue poesie sono in gran parte anonime, come per nascondere il suo io dietro i versi - era a disagio con chi lo indicava come attore principale della caduta del Muro di Berlino. Nel r99i chiese che il documento finale del Sinodo dei vescovi per l'Europa non menzionasse un suo «ruolo specifico" nei cambiamenti dell'Est. «È la Chiesa che ha contato in questo processo, non il papa», si giustificava ${ }^{68}$.

Il suo obiettivo era l'evoluzione pacifica e non la rivoluzione violenta, per richiamare la nota critica di Norwid al messianismo di Mickiewi$\mathrm{CZ}^{69}$. Ma il significato della transizione del $\mathrm{I} 989$ in Polonia andava ben oltre la Vistola. E lo storico Bronisław Geremek, attore di primo piano di quelle vicende, ha sostenuto che il 1989 aveva dato «il colpo di grazia alla rivoluzione francese del 1789 , alla lunga, bicentenaria storia della rivoluzione francese ${ }^{7^{\circ}}$. Ritorna in questa osservazione una distinzione, notata con finezza da Buttiglione: "Mentre in Occidente si fa strada, verso la fine dell'Ottocento, l'idea di una rivoluzione che sia una rottura assoluta con tutto il passato, la rivoluzione continua a essere pensata in Polonia come la resurrezione» ${ }^{7}$.

Si tratta di una distinzione sottile, difficile da cogliere non solo per gli Occidentali, ma anche per chi, in Polonia, trascorsi appena trenta anni, non apprezza la transizione del 1989 .

68 E. Mauro, P. Mieli, Così cambiammo il volto dell'Europa, «La Stampa» 4 marzo 1992.

69 Cfr. M. Masłowski, Problemy tożsamości. Szkice mickiewiczowskie i (post)romantyczne, Lublin 2006, p. 391.

70 Bronislaw Geremek en diálogo con Juan Carlos Vidal, Madrid I997, p. 206.

$7 \mathrm{I}$ R. Buttiglione, Il pensiero dell'uomo, op. cit., p. 42. 


\section{Bibliografia}

Accattoli L., Del Rio D., Wojtyta. Il nuovo Mosè, Milano 1988.

Atenagora, Clément O., Umanesimo spirituale. Dialoghi tra Oriente e Occidente, Cinisello Balsamo 2or3.

L'attività della Santa Sede nel 1979, Città del Vaticano 1980.

Brian P., Thy Kingdom Come: Patriotism, Prophecy, and the Catholic Hierarchy in Nineteenth-Century Poland, «The Catholic Historical Review» 2 (2003), p. 213-239.

Bronislaw Geremek en diálogo con Juan Carlos Vidal, Madrid I997.

Buttiglione R., Il pensiero dell'uomo che divenne Giovanni Paolo II, Milano I998.

Cazzago A., Giovanni Paolo II. «Ama gli altri popoli come il tuo», Milano 20I3.

Draguła A., Teologia narodu bez teologii, «Więź» 3 (2017), p. 226-232.

Ehrlich E., Remarks on Some Aspects of Messianism, transl. by K. Popowicz, "Theological Research" 7 (2019), p. 29-51.

From Solidarity to Freedom. International Conference, Warsaw-Gdañs, August 29-3I, 2005, Warsaw-Gdańsk 2005.

Giovanni Paolo II, Ai partecipanti ad un simposio internazionale su "Ivanov e la cultura del suo tempo», 28 maggio 1983, in: Insegnamenti di Giovanni Paolo II, Vol. 6/r: 1983, Città del Vaticano 1983, p. 1378-138r.

Giovanni Paolo II, Ai pellegrini polacchi, 23 ottobre 1978, in: Insegnamenti di Giovanni Paolo II, Vol. I: 1978, Città del Vaticano I979, p. 52.

Giovanni Paolo II, Ai rappresentanti dell'Istituto del patrimonio nazionale polacco, degli scienziati e dei cultori del poeta polacco Cyprian Kamil Norwid, $\mathrm{I}^{\circ}$ luglio 200I, in: Insegnamenti di Giovanni Paolo II, Vol. 24/2: 200I, Città del Vaticano 2002, p. 7-8.

Giovanni Paolo II, Al Corpo diplomatico, 20 ottobre 1978, in: Insegnamenti di Giovanni Paolo II, Vol. I: 1978, Città del Vaticano 1979, p. 27.

Giovanni Paolo II, All'Arcidiocesi di Cracovia, 23 ottobre 1978, in: Insegnamenti di Giovanni Paolo II, Vol. I: 1978, Città del Vaticano 1979, p. 56-57.

Giovanni Paolo II, L'allocuzione all'Organizzazione delle Nazioni Unite per l'Educazione, la Scienza e la Cultura, 2 giugno 1980, in: Insegnamenti di Giovanni Paolo II, Vol. 3/r: 1980, Città del Vaticano I98I, p. I636-I655.

Giovanni Paolo II, La celebrazione dei Vespri nella Heldenplatz, to settembre 1983, in: Insegnamenti di Giovanni Paolo II, Vol. 6/2: 1983, Città del Vaticano 1984 , p. 436-444.

Giovanni Paolo II, Dono e mistero. Nel $50^{\circ}$ del mio sacerdozio, Città del Vaticano 1996. 
Giovanni Paolo II, L'incontro con le autorità civili, 2 giugno I979, in: Insegnamenti di Giovanni Paolo II, Vol. 2/r: 1979, Città del Vaticano I979, p. I380.

Giovanni Paolo II, Lettera apostolica Rutilans Agmen alla Chiesa di polonia nel IX Centenario del martirio di san Stanislao, 8 maggio 1979, in: Insegnamenti di Giovanni Paolo II, Vol. 2/r: I979, Città del Vaticano I979, p. I095-IIor.

Giovanni Paolo II, Lettera enciclica «Slavorum apostoli», 2 giugno I985.

Giovanni Paolo II, Memoria e identità. Conversazioni a cavallo dei millenni, Milano 2005.

Giovanni Paolo II, L'omelia della messa a Le Bourget, I giugno I980, in: Insegnamenti di Giovanni Paolo II, Vol.3/r: 1980, Città del Vaticano I980, p. 1585-I592.

Giovanni Paolo II, L'omelia della messa a Lisieux, 2 giugno I980, in: Insegnamenti di Giovanni Paolo II, Vol. 3/r: I980, Città del Vaticano I980, p. I659.

Giovanni Paolo II, L'omelia sul colle dei martiri, 5 ottobre 1980, in: Insegnamenti di Giovanni Paolo II, Vol. 3/2: 1980, Città del Vaticano I981, p. 777.

Giudizio preliminare sullo svolgimento della visita di Giovanni Paolo II in Polonia (periodo 2-5 giugno 79), Archiwum Akt Nowych w Warszawie, Urząd do Spraw Wyznań w Warszawie, r37/r8, k. 62-68.

Impagliazzo M., Giovanni Paolo II e l'Italia, in: I cattolici e le Chiese cristiane durante il pontificato di Giovanni Paolo II (1978-2005), a cura di E. Guerriero, M. Impagliazzo, Cinisello Balsamo 2006, p. 97-I24.

Jan Paweł II, Pielgrzymki do Ojczyzny. Przemówienia, homilie, Kraków 2012.

Kemp-Welch A., Poland under Communism. A Cold War History, Cambridge 2008.

Kepel G., La Revanche de Dieu. Chrétiens, juifs et musulmans à la reconquête du monde, Paris I99I.

Kobyliński A., Le dimensioni etiche dell'odierna pentecostalizzazione del cristianesimo, «Rivista Teologica di Lugano» 20 (2015), p. 207-228.

Kula M., La storia «ad usum populi», «Limes» I (2014), p. 67-76.

Lecomte B., La verità prevarrà sempre sulla menzogna. Come papa Giovanni Paolo II ha sconfitto il comunismo, Milano 1992.

Marinelli L., Chi sono i polacchi?, «Limes» I (20I4), p. 57-65.

Masłowski M., Problemy tożsamości. Szkice mickiewiczowskie i (post)romantyczne, Lublin 2006.

Mauro E., Mieli P., Così cambiammo il volto dell'Europa, «La Stampa», 4 marzo I992, p. 2-3.

Michalski C., La religione di Smolensk, «Limes» I (20I4), p. 25-3I.

Michel P., Messianisme polonaise et histoire contemporaine, in: Le rêve de Compostelle. Vers la restauration d'une Europe chrétienne?, sous la direction de R. Luneau, P. Ladrière, Paris I989, p. 52-67. 
Mickiewicz A., Scritti politici, Torino 1965.

Mokrzycki M., B. Grysiak, I martedì di Karol. La vita quotidiana di Giovanni Paolo II, Cinisello Balsamo 2013.

Morozzo della Rocca R., Giovanni Paolo II papa slavo, «Sanctorum» 3 (2006), p. $55-59$.

Morozzo della Rocca R., Tra Est e Ovest. Agostino Casaroli diplomatico vaticano, Cinisello Balsamo 20I4.

Non abbiate paura! André Frossard dialoga con Giovanni Paolo II, Milano I983.

Norwid C. K., Rzecz o wolności stowa, in: C. K. Norwid, Pisma wszystkie, Warszawa i971, t. 3: Poematy, p. 557-623.

Norwid C. K., Znicestwienie Narodu, in: C. K. Norwid, Pisma wszystkie, t. 7: Proza, Warszawa i973, p. 85-92.

Plumyène J., Le nazioni romantiche. Storia del nazionalismo nel XIX secolo, Firenze 1982.

La politica del Vaticano nel momento attuale (progetto di intervento), Archiwum Akt Nowych w Warszawie, Urząd do Spraw Wyznań w Warszawie, 136/24, k. $16-25$.

Półtawska W., Diario di un'amicizia. La famiglia Póttawski e Karol Wojtyta, Cinisello Balsamo 2010, p. 403-404.

Raina P., Arcybiskup Dąbrowski. Rozmowy watykańskie, Warszawa $200 \mathrm{I}$.

Riccardi A., Giovanni Paolo. La biografia, Cinisello Balsamo 201 .

Riccardi A., Il secolo del martirio. I cristiani nel Novecento, Milano 2000.

Roccucci A., Senso della storia e tensione escatologica. Giovanni Paolo II e la santità di fronte alla crisi della modernità, «Sanctorum» 3 (2006), p. 15-30.

Rojek P., John Paul II and the Polish Messianism. Introduction to the Liturgy of History, transl. by K. Popowicz, "Theological Research" 7 (20I9), p. 9-27.

Rojek P., Liturgia dziejów. Jan Pawet II i polski mesjanizm, Kraków 2016.

Signifredi M., Cristo al servizio della Polonia, «Limes» 6 (2018), p. 235-242.

Signifredi M., Giovanni Paolo II e la fine del comunismo. La transizione in Polonia (1978-1989), Milano 2013.

Soviet Deliberations during the Polish Crisis, I980-I98I, ed. M. Kramer, Washington r999.

Tischner J., Guarire il lavoro ammalato, in: J. Tischner, Etica della solidarietà $e$ del lavoro, trad. A. Setola, Castel Bolognese 2010, p. I42-I47.

Walicki A., Adam Mickiewicz and Polish Romantic Messianism, «Dialogue and Universalism» 3-4 (2000), p. I65-197.

Walicki A., Philosophy and Romatic Nationalism: The Case of Poland, Oxford I982.

Wierzbicki A., Kruche dziedzictwo. Jan Pawet II od nowa, Warszawa 2018. 
Wierzbicki A. M., Present Condition and Role of the Catholic Church in Poland, "Occasional Papers on Religion in Eastern Europe» 38 (2018), p. 92-103.

Wojtyła K., Alle fonti del rinnovamento. Studio sull' attuazione del Concilio Vaticano Secondo, Città del Vaticano, 1981.

Wojtyła K., Le poesie giovanili (Salterio di Davide, Libro slavo, Salterio rinascimentale), Roma 2004.

Wojtyła K., Il Teatro Rapsodico. Articoli e Lettere, Roma 2003.

Wojtyła K., Tutte le opere letterarie. Poesie, drammi e scritti sul teatro, MilanoCittà del Vaticano 200I. 